\section{La biología y las matemáticas}

\author{
Autor: \\ iD Francisco Javier León ${ }^{1}$ \\ ${ }^{1}$ Profesor Asociado Universidad de Santander, Calle 70 \# 55-210 \\ Campus lagos del cacique, Facultad de Ciencias exactas, físicas \\ y naturales, Bucaramanga, Colombia, fleon@udes.edu.co
}

\section{DOl:}

http://dx.doi.org/10.15649/2346075X.445

Los hallazgos de Mendel y sus leyes ${ }^{1}$ han trascendido en la comprensión de la Biología y la Medicina, dicho trabajo abrió las puertas para que otros científicos como Hardy y Weinberg demostraran que las matemáticas tienen un rol fundamental en la comprensión de distintos fenómenos biológicos y de esta forma el equilibrio en las frecuencias genotípicas y alélicas ${ }^{2}$ es más que el binomio cuadrado perfecto. Mediante las matemáticas se percibe que, tras cierto número de generaciones en una población de tamaño infinito se logra un equilibrio genético siempre y cuando se cumplan algunas condiciones como el apareamiento aleatorio, ausencia del efecto fundador, sin deriva genética, entre otros; siendo lo anterior fundamental para comprender diferentes fenómenos genéticos relacionados con la herencia y la genética poblacional. Años más tarde, Fisher empleó las matemáticas para combinar las leyes de Mendel con la selección natural de Darwin y de esta forma plantear su propia teoría evolutiva.

Como los ejemplos anteriores, existen otros trabajos donde la biología celular y molecular, la medicina, las ingenierías, la economía, la agronomía se robustecen con el poder que tienen las matemáticas para comprender los fenómenos biológicos relacionados con cambios en secuencias de $\mathrm{ADN}^{3}$, polimorfismos genéticos $^{4}$, en proteínas ${ }^{5}$, en la fisiología celular, en un organismo o en una comunidad. Es por ello que cada día la estadística aplicada tiene impacto a nivel clínico, social y de salud pública pues fortalece diversas áreas del conocimiento que se apoyan en un sin número de algoritmos matemáticos y abre la posibilidad para plantear diseños de experimentos, análisis e interpretación de datos, comprender la magnitud de los errores y la toma de decisiones frente a los datos que

\section{EDITORIAL}

se generan, puesto que cuantifican y cualifican imparcialmente los resultados en la investigación. ${ }^{6,7}$

\section{REFERENCIAS}

1. Allen, G. E. (2017). La ciencia de la vida en el siglo XX. Fondo de Cultura Económica.

2. Hu, X., Duan, X., Pan, D. et al. J Syst Sci Complex (2017) 30: 101. https://doi.org/10.1007/s11424017-6187-4

3. Burguillo Muñoz, F. J. (2015). Desarrollo de un algoritmo de Mínimos Cuadrados Parciales para análisis de datos de chips de ADN usando el estadístico VIP para selección de genes y clasificación binaria.

4. Mantilla, G., Castillo, A., Pérez, V. L., Vargas, C. I., \& Rondón, F. (2017). S19W polymorphism (Ser19Ter) of APOA5 and its relationship with hypertriglyceridemia in a Colombian population. Revista de la Universidad Industrial de Santander. Salud, 49(1), 29-35.

5. Rodríguez-Piñeiro, A. M., Páez de la Cadena, M., \& Rodríguez-Berrocal, F. J. (2008). La inevitable estadística... en el mundo de la proteómica.

6. Tanur, J. M., Mosteller, F., Kruskal, W. H., Lehmann, E. L., Link, R. F., Pieters, R. S., \& RISING, G. (1992). La Estadística: Una guía de lo desconocido. Alianza.

7. Wilches, O. M. (1995). Métodos estadísticos multivariados en biología molecular y su aplicación en investigación agrícola. Agronomía colombiana, 12(1), 66-71. 